\title{
CURVATURE PINCHING FOR THREE-DIMENSIONAL MINIMAL SUBMANIFOLDS IN A SPHERE
}

\author{
YI-BING SHEN
}

(Communicated by Jonathan M. Rosenberg)

\begin{abstract}
In this paper, some pinching theorems for the Ricci curvature and the scalar curvature of three-dimensional compact minimal submanifolds in a sphere are given.
\end{abstract}

\section{INTRODUCTION}

Let $M^{n}$ be an $n$-dimensional compact orientable minimal submanifold in a unit $(n+p)$-sphere $S^{n+p}$. In [2] it was proved that if $n \geq 4$ and the Ricci curvature of $M^{n}$ is larger than $n-2$, then $M^{n}$ is totally geodesic in $S^{n+p}$. Recently, the corresponding problem for the three-dimensional case was treated in [4]. The aim of this paper is to improve the result of [4] so that the theorem of [2] is valid for the case $n=3$. Precisely, we prove

Theorem 1. Let $M^{3}$ be a three-dimensional compact minimal submanifold in a unit sphere $S^{3+p}$. If the Ricci curvature of $M^{3}$ is larger than 1 then $M^{3}$ is totally geodesic in $S^{3+p}$.

Moreover, for lower codimension, we have

Theorem 2. Let $M^{3}$ be a compact orientable minimal submanifold in $S^{3+p}$ with $p \leq 2$. If the Ricci curvature of $M^{3}$ is not less than $(5 p-4) /(4 p-2)$ then $M^{3}$ is totally geodesic in $S^{3+p}$.

In the same way as in the proof of Theorem 1, we also obtain

Theorem 3. Let $M^{3}$ be a compact minimal submanifold in $S^{3+p}$. If the scalar curvature of $M^{3}$ is larger than 4 then $M^{3}$ is totally geodesic.

Throughout this paper, all the manifolds dealt with are smooth and connected.

\section{Preliminaries}

In this section we state some notations and basic formulas. More details can be found in [4]. Let $M^{3}$ be a three-dimensional compact Riemannian manifold

Received by the editors November 19, 1990 and, in revised form, December 17, 1990.

1991 Mathematics Subject Classification. Primary 53C40; Secondary 53C42, 53C20.

Project supported by NNSFC. 
that is minimally immersed in a unit $(3+p)$-sphere $S^{3+p}$. We choose a local field of orthonormal frames $e_{1}, \ldots, e_{3+p}$ in $S^{3+p}$ such that, restricted to $M^{3}$, the vectors $e_{1}, e_{2}$, and $e_{3}$ are tangent to $M^{3}$. Unless otherwise stated, we agree on the following ranges of indices: $1 \leq i, j, k, \cdots \leq 3 ; 4 \leq \alpha, \beta, \cdots \leq 3+p$. The second fundamental form of $M^{3}$ in $S^{3+p}$ is

$$
\sigma=\sum_{\alpha, i, j} h_{i j}^{\alpha} \omega^{i} \otimes \omega^{j} \otimes e_{\alpha},
$$

of which the length square is $\|\sigma\|^{2}=\sum_{\alpha, i, j}\left(h_{i j}^{\alpha}\right)^{2}$.

Let $U M \rightarrow M^{3}$ be the unit tangent bundle over $M^{3}$. We define a function $f: U M \rightarrow \mathbf{R}$ by

$$
f(u)=\|\sigma(u, u)\|^{2}=\sum_{\alpha}\left(\sum_{i, j} h_{i j}^{\alpha} u^{i} u^{j}\right)^{2}
$$

for $u=\sum_{i} u^{i} e_{i} \in U M$. Since $U M$ is compact, $f$ attains its maximum at a vector in $U M$. Suppose that this vector is $v \in U M_{x_{0}}$ for some point $x_{0} \in M^{3}$. By taking $e_{1}=v$ at $x_{0}$ and letting

$$
b_{i j}=\sum_{\alpha} h_{11}^{\alpha} h_{i j}^{\alpha}
$$

from the maximality of $f$ we can choose vectors $e_{2}$ and $e_{3}$ at $x_{0}$ such that (cf. [4])

$$
\begin{gathered}
f(v)=b_{11}=\max _{u \in U M}\left\{\|\sigma(u, u)\|^{2}\right\}, \\
b_{i j}=0 \quad(i \neq j), \\
2 \sum_{\alpha}\left(h_{1 k}^{\alpha}\right)^{2}+b_{k k}-b_{11} \leq 0 \quad(k \neq 1), \\
\sum_{\alpha}\left(h_{11 i}^{\alpha}\right)^{2}+\sum_{\alpha} h_{11}^{\alpha} h_{11 i i}^{\alpha} \leq 0
\end{gathered}
$$

at the point $x_{0}$.

The Gauss equation of $M^{3}$ is

$$
R_{i j k l}=\delta_{i k} \delta_{j l}-\delta_{i l} \delta_{j k}+\sum_{\alpha}\left(h_{i k}^{\alpha} h_{j l}^{\alpha}-h_{i l}^{\alpha} h_{j k}^{\alpha}\right),
$$

from which and the minimality it follows that

$$
R_{i j}=2 \delta_{i j}-\sum_{\alpha, k} h_{i k}^{\alpha} h_{j k}^{\alpha}
$$

and

$$
R=6-\|\sigma\|^{2}
$$

where $R_{i j k l}, R_{i j}$, and $R$ denote the curvature tensor, the Ricci tensor, and the scalar curvature of $M^{3}$, respectively.

Summing up for $i$ in (2.7) and using (2.5) and the Ricci identity, we easily get [4]

(2.11) $0 \geq 3 f(v)+2 \sum_{\alpha, k \neq 1} b_{k k}\left(h_{1 k}^{\alpha}\right)^{2}-2 f(v) \sum_{\alpha, k \neq 1}\left(h_{1 k}^{\alpha}\right)^{2}-\sum_{k \neq 1}\left(b_{k k}\right)^{2}-f(v) b_{11}$

at the point $x_{0}$. 
Finally, as is well known, the curvature tensor of a three-dimensional manifold can be expressed as

$$
R_{i j k l}=\delta_{i k} R_{j l}-\delta_{i l} R_{j k}+\delta_{j l} R_{i k}-\delta_{j k} R_{i l}-\frac{1}{2} R\left(\delta_{i k} \delta_{j l}-\delta_{i l} \delta_{j k}\right) .
$$

\section{Proofs of Theorems 1 and 3}

We restrict ourselves to the point $x_{0}$ where the function $f$ defined by (2.2) attains its maximum. Then, from (2.3) and (2.4) one can easily see that

$$
\left(b_{k k}\right)^{2} \leq\left(\sum_{\alpha}\left(h_{11}^{\alpha}\right)^{2}\right)\left(\sum_{\alpha}\left(h_{k k}^{\alpha}\right)^{2}\right) \leq\left(b_{11}\right)^{2}
$$

for $k \neq 1$, from which and the three-dimensional minimality it follows that

$$
b_{22} \leq 0, \quad b_{33} \leq 0, \quad \sum_{k \neq 1}\left(b_{k k}\right)^{2} \leq\left(\sum_{k \neq 1} b_{k k}\right)^{2}=\left(b_{11}\right)^{2} .
$$

From (2.3) and (2.9) we have

$$
-\sum_{\alpha, k \neq 1}\left(h_{1 k}^{\alpha}\right)^{2}=R_{11}-2+b_{11}
$$

Substituting (3.3) into (2.11) and using (3.2), one can obtain

$$
\begin{aligned}
0 & \geq 3 f(v)+2 \sum_{\alpha, k \neq 1} b_{k k}\left(h_{1 k}^{\alpha}\right)^{2}+2 f(v)\left(R_{11}-2+b_{11}\right)-\sum_{k \neq 1}\left(b_{k k}\right)^{2}-f(v) b_{11} \\
& =-f(v)+2 \sum_{\alpha, k \neq 1} b_{k k}\left(h_{1 k}^{\alpha}\right)^{2}+2 f(v) R_{11}+f(v) b_{11}-\sum_{k \neq 1}\left(b_{k k}\right)^{2} \\
& \geq-f(v)+2 \sum_{\alpha, k \neq 1} b_{k k}\left(h_{1 k}^{\alpha}\right)^{2}+2 f(v) R_{11} .
\end{aligned}
$$

On the other hand, by (3.2), (2.6), and (3.1), we have respectively

$$
\sum_{\alpha, k \neq 1} b_{k k}\left(h_{1 k}^{\alpha}\right)^{2} \geq \frac{1}{2} \sum_{k \neq 1} b_{k k}\left(b_{11}-b_{k k}\right)=-\frac{1}{2} \sum_{i}\left(b_{i i}\right)^{2}
$$

and

$$
\sum_{\alpha, k \neq 1} b_{k k}\left(h_{1 k}^{\alpha}\right)^{2} \geq-f(v) \sum_{\alpha, k \neq 1}\left(h_{1 k}^{\alpha}\right)^{2}=f(v)\left(R_{11}-2+b_{11}\right) .
$$

Introducing (3.5) and (3.6) into (3.4), we get

$$
\begin{aligned}
0 & \geq-f(v)+2 f(v) R_{11}+f(v)\left(R_{11}-2\right)+\frac{1}{2}\left[\left(b_{11}\right)^{2}-\sum_{k \neq 1}\left(b_{k k}\right)^{2}\right] \\
& \geq 3 f(v)\left(R_{11}-1\right) .
\end{aligned}
$$

Thus, if the Ricci curvature of $M^{3}$ is larger than 1 then (3.7) implies that $f(v)=0$, i.e., $\|\sigma\|^{2}$ vanishes identically. This proves Theorem 1 . 
In the similar manner, it follows from (2.11), (3.5), (3.6), and (3.1) that

$$
\begin{aligned}
0 \geq & 3 f(v)+\left(\sum_{\alpha, k \neq 1} b_{k k}\left(h_{1 k}^{\alpha}\right)^{2}-2 f(v) \sum_{\alpha, k \neq 1}\left(h_{1 k}^{\alpha}\right)^{2}\right) \\
& +\left(\sum_{\alpha, k \neq 1} b_{k k}\left(h_{1 k}^{\alpha}\right)^{2}-\sum_{k \neq 1}\left(b_{k k}\right)^{2}-\left(b_{11}\right)^{2}\right) \\
\geq & 3 f(v)-3 f(v) \sum_{\alpha, k \neq 1}\left(h_{2 k}^{\alpha}\right)^{2}-\frac{3}{2} f(v) b_{11}-\frac{3}{2} \sum_{k \neq 1}\left(b_{k k}\right)^{2} \\
\geq & \frac{3}{2} f(v)\left\{2-\sum_{\alpha, i}\left(h_{i i}^{\alpha}\right)^{2}-2 \sum_{\alpha, k \neq 1}\left(h_{2 k}^{\alpha}\right)^{2}\right\} \\
\geq & \frac{3}{2} f(v)\left\{2-\|\sigma\|^{2}\left(x_{0}\right)\right\} .
\end{aligned}
$$

Thus, if the scalar curvature of $M^{3}$ is larger than 4 , i.e., $\|\sigma\|^{2}<2$, then (3.8) implies that $f(v)=0$, i.e., $M^{3}$ is totally geodesic. Theorem 3 is proved.

\section{Proof of Theorem 2}

For a compact orientable minimal submanifold $M^{3}$ in $S^{3+p}$, a standard calculation gives (cf. [4, Lemma 1.2])

$$
\int_{M^{3}}\left\{2 \sum_{\alpha, i, j, k, l} h_{i j}^{\alpha}\left(h_{k l}^{\alpha} R_{l i j k}+h_{i l}^{\alpha} R_{l k j k}\right)+\frac{1}{p}\|\sigma\|^{4}-3\|\sigma\|^{2}\right\} * 1 \leq 0,
$$

where ${ }^{*} 1$ denotes the volume element of $M^{3}$.

Let $Q(x)$ be the function assigns to each point $x$ of $M^{3}$ the minimum of the Ricci curvatures of $M^{3}$ at that point $x$. For each $\alpha$, let $\alpha_{i}$ be the eigenvalues of the matrix $\left(h_{i j}^{\alpha}\right)$. Then, by $(2.12)$ we have

$$
\begin{aligned}
\sum_{i, j, k, l} h_{i j}^{\alpha}\left(h_{k l}^{\alpha} R_{l i j k}+h_{i l}^{\alpha} R_{l k j k}\right) & =\sum_{i \neq j}\left(\alpha_{i}^{2}-\alpha_{i} \alpha_{j}\right)\left(R_{i i}+R_{j j}-\frac{1}{2} R\right) \\
& =3 \sum_{i} \alpha_{i}^{2} R_{i i}-\frac{1}{2} R \sum_{i} \alpha_{i}^{2} \geq\left(3 Q-\frac{1}{2} R\right) \sum_{i, j}\left(h_{i j}^{\alpha}\right)^{2},
\end{aligned}
$$

from which and (4.1) it follows that

$$
\int_{M^{3}}\|\sigma\|^{2}\left(6 Q-R+\frac{1}{p}\|\sigma\|^{2}-3\right)^{*} 1 \leq 0,
$$

i.e., by (2.10),

$$
\int_{M^{3}}\|\sigma\|^{2}\left(6 Q-9+\frac{p+1}{p}\|\sigma\|^{2}\right){ }^{*} 1 \leq 0 .
$$

On the other hand, the well-known Simons inequality [5] for $n=3$ is

$$
\int_{M^{3}}\|\sigma\|^{2}\left(\frac{3 p}{2 p-1}-\|\sigma\|^{2}\right)^{*} 1 \leq-\int_{M^{3}}\|\nabla \sigma\|^{2 *} 1 \leq 0,
$$


from which and (4.2) we get

$$
\int_{M^{3}}\|\sigma\|^{2}\left(Q-\frac{5 p-4}{4 p-2}\right){ }^{*} 1 \leq 0 .
$$

Thus, if $Q>(5 p-4) /(4 p-2)$, then (4.4) implies that $\|\sigma\|^{2}=0$ identically. We now consider the case that $Q=(5 p-4) /(4 p-2)$. Then, (4.2) becomes

$$
\int_{M^{3}}\|\sigma\|^{2}\left(\|\sigma\|^{2}-\frac{3 p}{2 p-1}\right){ }^{*} 1 \leq 0,
$$

which together with (4.3) gives that $\nabla \sigma=0$, and hence, since $\|\sigma\|^{2}$ is constant, $\|\sigma\|^{2}=0$ or $3 p /(2 p-1)$. Since the Ricci curvature of $M^{3}$ is positive everywhere, $M^{3}$ cannot be the Clifford hypersurface. Now, Theorem 2 follows directly from the well-known result of [1] for $n=3$.

Remark. It is clear that the pinching values given here are not the best possible. In general, for each pair $(n, p)$, there is a best pinching value for minimal $M^{n}$ in $S^{n+p}$. Really, in [2] the pinching constant $n-2$ for the Ricci curvature is not sharp for $n \neq 4$ and $p \neq 1$. In [3], it was proved that there exists an isometric minimal immersion of $S_{1 / 8}^{3}$ into $S^{9}$, where $S_{1 / 8}^{3}$ denotes the 3-sphere with constant sectional curvature $1 / 8$. On the other hand, it is well known that every three-dimensional Einstein manifold is of constant curvature. So, perhaps one can surmise that the best possible pinching value of the Ricci curvature for minimal $M^{3}$ in $S^{3+p}$ would be $\frac{1}{4}$. However, we have not demonstrated it.

\section{ACKNOWLEDGMENT}

The author would like to thank Professor J. Eells and ICTP for their hospitality during participating in the College on Differential Geometry 1989. The author would also like to thank the referee for many helpful suggestions.

\section{REFERENCES}

1. S. S. Chern, M. do Carmo, and S. Kobayashi, Minimal submanifolds of a sphere with second fundamental form of constant length, Fund. Anal. Rel. Fields, Springer-Verlag, (1970), 5975.

2. N. Ejiri, Compact minimal submanifolds of a sphere with positive Ricci curvature, J. Math. Soc. Japan 31 (1979), 251-256.

3. K. Mashimo, Minimal immersions of 3-dimensional sphere into spheres, Osaka J. Math. 21 (1984), 721-732.

4. Y. B. Shen, On intrinsic rigidity for minimal submanifolds in a sphere, Science in China, Ser. A 32 (1989), 769-781.

5. J. Simons, Minimal varieties in Riemannian manifolds, Ann. of Math. (2) 88 (1968), 62105.

Department of Mathematics, Hangzhou University, Hangzhou 310028, People's RePUBLIC OF CHINA 